\title{
Joint-Targeting Drug Delivery System for Rheumatoid Arthritis: siRNA Encapsulated Liposome
}

\author{
Yukiko Komano ${ }^{1^{*}}$, Nobuhiro Yagi $^{2}$ and Toshihiro Nanki ${ }^{3}$ \\ ${ }^{1}$ Jyujyo Takeda Rehabilitation Hospital. 32 Hattanda, Kisyoin, Japan \\ ${ }^{2}$ Kyowa Hakko Kirin Co., Ltd. 3-6-6 Asahimachi, Machida-shi, Tokyo, Japan \\ ${ }^{3}$ Department of Clinical Research Medicine, Teikyo University, Tokyo, Japan
}

*Corresponding author: Toshihiro Nanki, Division of Rheumatology, Department of Internal Medicine Toho University School of Medicine, Tokyo, Japan, Tel: +81-3-3762-4151; Fax: +81-3-3298-0045; Email: toshihiro.nanki@med.toho-u.ac.jp

Received date: February 08, 2015, Accepted date: April 12, 2015, Published date: April 19, 2015

Copyright: @ 2015 Komano $\mathrm{Y}$, et al. This is an open-access article distributed under the terms of the Creative Commons Attribution License, which permits unrestricted use, distribution, and reproduction in any medium, provided the original author and source are credited.

\begin{abstract}
Rheumatoid Arthritis (RA) is characterized by persistent inflammation in multiple joints. Uncontrolled active RA causes disability, decreases quality of life, and increases comorbidity. Over the past two decades, the importance of aggressive treatment as early as possible has been emphasized to improve outcomes, and, most importantly, inhibit the destruction of joints. RNA interference, mediated by small interfering RNA (siRNA), is a powerful method used to silence genes with a high degree of specificity. Developing a means to deliver siRNA via a systemic injection to multiple affected joints is of importance for applying this technology to the treatment of RA. A recent study showed that a wrapsome (WS), which was designed with a core composed of a cationic lipid bilayer and siRNA complex enveloped in a neutral lipid bilayer with polyethylene glycol on the surface, could be a potential vehicle for siRNA delivery for the treatment of arthritis. The complex of siRNA and WS (siRNA/WS) selectively accumulated in the inflamed synovium. Furthermore, treatment with siRNA-targeting tumor necrosis factor-a/WS ameliorated arthritis in a murine model. In this manuscript, we review potential of siRNA as a therapeutic tool for rheumatoid arthritis.
\end{abstract}

Keywords: Rheumatoid arthritis; RNA interference; Small interfering RNA; Drug delivery

\section{Introduction}

Rheumatoid Arthritis (RA) is a chronic systemic autoimmune inflammatory disease affecting around $1 \%$ of the adult population [1]. RA not only causes persistent arthritic pain, but also significant joint destruction with disability. The main pathology of RA is polyarticular inflammation along with hyperplasia of the synovial lining cells and pannus formation. Synovial hyperplasia is characterized as tumor-like proliferation and is thought to be a major cause of RA joint damage.

Historically, symptom reductions using nonsteroidal antiinflammatory drugs and analgesics dominated RA management. However, marked changes have occurred in RA therapy over the past two decades. The importance of the early use of disease-modifying antirheumatic drugs (DMARDs) has been emphasized. DMARDs, represented by methotrexate, have been shown to reduce synovitis, systemic inflammation, and disability. The introduction of biologic agents has revolutionized the outcome of RA patients, making clinical remission or low disease activity possible as a standard goal in daily practice. Biologic drugs that selectively block the function of cell surface molecules or inflammatory cytokines, including tumor necrosis factor- $\alpha$ (TNF- $\alpha$ ), are also available for RA treatment.

In spite of these advances, the higher risk of serious infections including tuberculosis following the administration of biologic drugs has been a major concern, and limits the indication of their use especially for those with multiple comorbidities [2,3]. In addition, there are still patients who do not respond to these biologic agents [4]. The high price of biologics also limits their availability, and the cost- effectiveness of using them is a matter of debate. Thus, new and better treatment strategies are needed for RA.

\section{Knocking down undruggable target proteins using synthetic small interfering RNA}

Human genome sequence analysis has revealed over 20,000 protein species that may act as therapeutic targets [5]. Biological and medical science has provided numerous insights; however, many of their therapeutic targets cannot be applied to conventional pharmacological strategies, such as synthetic drug molecule or antibody antagonists and agonists [6]. In order to down regulate the above undruggable proteins as therapeutic targets, RNA interference (RNAi) has been considered as a promising new technology and has also become a next-generation drug candidate. RNAi has been induced by double-stranded short sequenced RNA called small interfering RNA (siRNA), which has a complemented sequence with a targeted gene mRNA transcript [7]. siRNA is incorporated with an RNA-induced silencing complex, and then, inhibits gene expression by degrading the target mRNA, and RNAi technology has become a powerful and common tool for gene knock down in vitro.

\section{Wrapsome (WS), a novel lipid nanoparticle encapsulating siRNA}

A promising strategy to treat RA with nucleic acids may be to use long-circulating and stabilized particle formulations selectively delivering siRNA into multiple inflammatory joints [8]. This strategy may be the knock down of any gene of interest involved in developing arthritis, including intracellular protein targets that are unable to inhibit by monoclonal antibodies (mAbs) such as transcription and 
nuclear factors. The key for the successful treatment of RA with siRNA will be to obtain a safe and efficient siRNA delivery vehicle that selectively accumulates in inflammatory joints by phenomenon known as the enhanced permeability and retention (EPR) effect [9]. The EPR effect results from the extravasation of long-circulating and high molecular weight materials through leaky vasculature of cancer and inflammatory tissues. Wrapsome (WS) is a recently developed lipid nanoparticle that has been specifically designed to deliver siRNA into inflammatory sites [10]. WS has a core comprised of cationic lipid particles and a siRNA complex (Figure 1). This cationic complex may be helpful in transfecting siRNA into the cytosol of the targeted cell. Because the cationic core complex of WS is covered with a neutral lipid bilayer and polyethylene glycol (PEG), siRNA is located on the inside of the particle and none is on the surface. Furthermore, the neutral and PEG-modified surface helps WS to escape the recognition by reticuloendothelial system (RES) and obtain a long half-life [10]. Thus, WS formulations are promising for the selective delivery of siRNA into inflammatory joints via the EPR effect, and subsequent knockdown of targeted genes involved in RA.

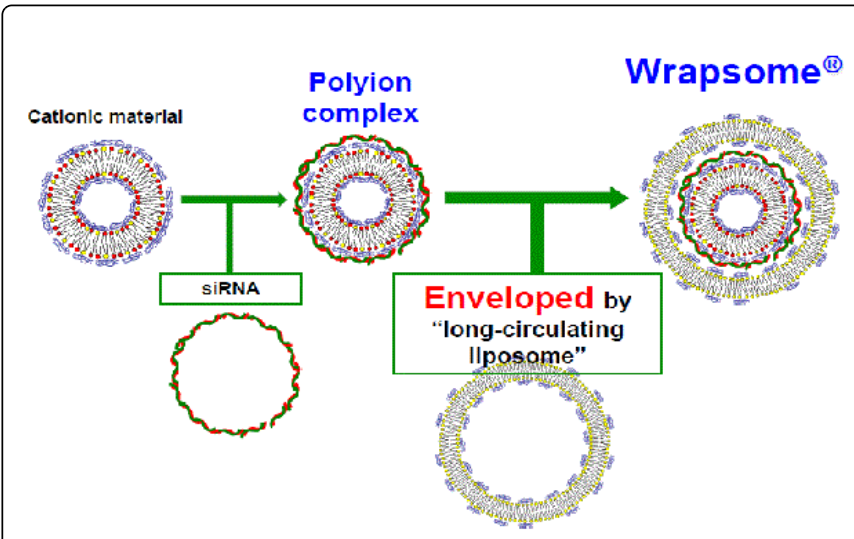

Figure 1: Structure of Wrapsome. Wrapsome designed to have envelope on the surface which made with long-circulating liposome, and polyion complex in inside. Envelope allows siRNA to circulate systemically and accumulate into synovial joint. Inner polyion complex is similar in structure to lipid-based transfection agent and considered to accommodate cellular transduction of siRNA.

\section{Knocking down TNF- $a$ using the complex of siRNA and WS (siRNA/WS) in mice arthritis}

To explore the therapeutic potential of systemically administered siRNA for RA, we tested the complex of siRNA and WS (siRNA/WS) in mice with collagen-induced arthritis (CIA) [11]. The accumulation of siRNA/WS was observed in the inflamed joints of CIA mice that were intravenously injected with Cy5-labeled siRNA/WS. The intensity of the fluorescence level remained high in the arthritic joints up to $48 \mathrm{hrs}$ post-injection. The fluorescence intensity was higher in synovial cells than in splenocytes, bone marrow cells, or peripheral blood leukocytes. The majority of Cy5-positive synovial cells were $\mathrm{CD} 11 \mathrm{~b}+$ macrophages and neutrophils with only a few CD3+ T cells. The severity of arthritis and TNF- $\alpha$ mRNA levels in the joints was significantly less with TNF- $\alpha$ siRNA/WS than with control siRNA/WS. These results suggest that both the selective delivery and retention of Cy5-siRNA to the inflamed synovium could be achieved using WS as a carrier of siRNA, and that systemically administered siRNA/WS might efficiently target macrophages and neutrophils in inflamed joints. Systemically administered TNF- $\alpha$-targeting siRNA/WS could also inhibit CIA through the down-regulation of TNF- $\alpha$ expression in synovial tissue. The siRNA/WS based therapy has some limitations that need to be overcome to before clinically applied for RA. First, although the treatment with TNF- $\alpha$ siRNA/WS from the day when clinical arthritis just started developing showed therapeutic effect in mouse CIA model, the treatment was ineffective against advanced arthritic mice. In order to gain clinical effect through knocking down, we need to find the optimal timing for administration of the siRNA/WS. We might consider that pathophysiologic role of a targeted protein could be different in phases (i.e. acute or chronic) of a disease. Combining siRNA against different targeting molecule may bring out robust therapeutic efficacy of this system. Secondary, less frequent injection schedule need to be explored for human chronic diseases such as rheumatoid arthritis. In our mouse CIA model, systemic injection of WS/siRNA against TNF- $\alpha$ three times per week was needed to lower the disease severity.

\section{Potency (advantage) of the therapeutic usage of siRNA/WS against rheumatoid arthritis}

Only a few studies have reported the success of siRNA-based therapy for RA in animal models (Table 1) [12-15]. Successful injection of siRNA directly into the affected joints was reported $[14,15]$. However, local injection is not a proper method for multiple joint inflammation. Khoury et al. [12] reported that systemic administration of a cationic liposome carrying siRNA directed against TNF- $\alpha$ or a cocktail of siRNAs directed against pro-inflammatory cytokines, including interleukin (IL) $-1,-6$, and -18 , was effective against CIA. However, the cationic liposome used in the above studies could be rapidly distributed to the RES due to its micron-sized diameter and positive surface property.

One of the distinct properties of siRNA/WS is to be nano-sized (100 $\mathrm{nm}$ in diameter) and neutrally charged, resulting in its efficient distribution into peripherally inflamed tissue [10]. We confirmed the marked accumulation and cellular incorporation of siRNA encapsulated in WS in the inflamed synovium. In addition, siRNA/WS was shown to be selectively incorporated into macrophages and neutrophils in the synovium. The above properties of siRNA/WS may enable us to develop tissue/molecular-specific targeting therapies (Figure 2).

Synovial macrophages play central roles in the pathogenesis of RA by producing a number of pro-inflammatory cytokines such as TNF- $\alpha$, IL-1, and IL-6 [16]. In addition, macrophages produce chemokines, including CC chemokine ligand (CCL)3, CCL5, CXC chemokine ligand 8 , and $\mathrm{CX} 3 \mathrm{C}$ chemokine ligand 1 , which also mediate monocyte migration into the synovium, and stimulate angiogenesis [17]. Metalloproteases are also produced by synovial macrophages, which contribute to joint tissue degradation [18]. Cell-cell interactions between macrophages and other synovial cells including fibroblasts are also critical for the resulting inflammation and tissue damage [19]. Various molecules expressed by synovial macrophages could be targeted using siRNA/WS [11]. Furthermore, targeted inhibition of proinflammatory signal transduction pathways (i.e. nuclear factorkappa B) in macrophages could be possible by siRNA/WS [20]. 
Page 3 of 5

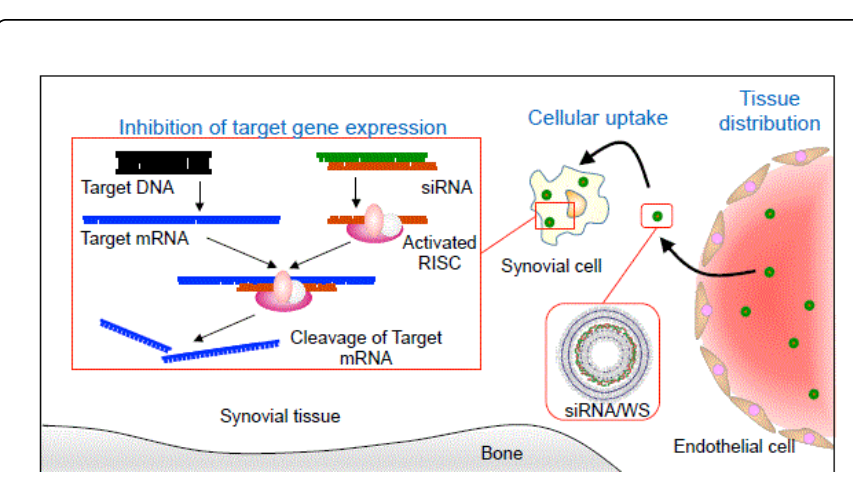

Figure 2: siRNA delivery via Wrapsome and inhibition of target gene expression in inflamed synovia siRNA encapsulated Wrapsome circulates and leaks out into synovial tissue from blood vessel located in inflamed synovia by EPR effect after intravenously administration. Target gene expressing cell such as macrophage incorporates siRNA encapsulated Wrapsome in synovial tissue. siRNA inhibits target gene expression with RNA interference pathway.

A number of studies have suggested that neutrophils contribute to the pathogenesis of RA [21]. Neutrophils are the most abundant cell present in the synovial fluid of affected joints and are also abundant at the pannus/cartilage junction, in which most tissue damage occurs [22]. Neutrophils isolated from RA joints have an activated phenotype, which indicates that they have undergone an activation process and have likely released important inflammatory mediators [23]. The role of neutrophils has also been tested in experimental models of autoimmune arthritis. In the $\mathrm{K} / \mathrm{B} \times \mathrm{N}$ mouse $\mathrm{RA}$ model, the transfer of autoreactive immunoglobulin from the $\mathrm{K} / \mathrm{B} \times \mathrm{N}$ mouse into mice induced an inflammatory response reminiscent of human RA [24]. Using this model, Wipke BT et al. showed that treatment with a neutrophil-depleting $\mathrm{mAb}$ before and following the transfer of arthritogenic serum completely blocked joint-specific inflammation in $\mathrm{K} / \mathrm{B} \times \mathrm{N}$ serum recipient mice, which indicated an indispensable role for neutrophils in disease initiation [25]. Although extracorporeal granulocytapheresis was previously shown to be effective in a subset of patients with RA [26,27], an anti-rheumatic drug that specifically targets neutrophils has not yet been identified. Using siRNA/WS, it may be possible to develop RA treatment by targeting neutrophils recruited to arthritic joints. Since the expression of $\mathrm{FcR} \gamma, \mathrm{C} 5 \mathrm{aR}$, and CD11a/LFA-1 is required on neutrophils for the induction of inflammatory arthritis [28], these molecules may be targets by siRNA/WS.

siRNA/WS could potentially be applied to various diseases other than RA. These may include inflammatory diseases such as antineutrophil cytoplasmic autoantibody-associated vasculitis and psoriasis, in which neutrophils play an important role in pathogenesis, and other diseases including macrophage activation syndromes [29-31]. Understanding the molecular mechanism of siRNA/WS delivery should help us further explore the therapeutic potency of WSmediated siRNA delivery.

\section{Limitations of siRNA and nanoparticle-based in vivo knock down systems}

A systemically administered siRNA vehicle has not yet been used clinically in spite of the technological advances that have recently taken place in siRNA therapies. This may be due to several remaining issues regarding siRNA or nanoparticle-based drug delivery vehicles.

RNA stability was the first issue to be solved. siRNA easily undergoes enzymatic digestion and is cleared from the circulation within several minutes of being intravenously injected [32]. Chemical modifications of the nucleotide and phosphate backbone were shown to be effective in protecting siRNA from enzymatic digestion [33]. Chemically modified siRNAs have been entered into clinical trials for age-related macular degeneration [34] and acute kidney injuries [35]. Because of its size and water solubility, siRNA is also easily excreted in the urine even if it is stabilized with chemical modifications [36]. Therefore, topical use or a kidney excretion pathway-related target would be suitable for naked siRNA molecules as pharmaceuticals. On the other hand, ion complex formation with cationic materials or its inclusion onto particles also stabilizes siRNA under physiological conditions [36]. Cationic materials have been used to form ion complexes with siRNA and also help with its transfection into the cytosol after siRNA reaches its target cell. These cation-complexed type formulations have been used for in vivo transfection and mainly target the liver, spleen and lung because RES organs trap foreign materials [37]. Several clinical tests are ongoing for liver cancer and hepatic diseases $[38,39]$.

The second issue was improving targeted tissue distributions of the siRNA. Difficulties have been reported for the selective delivery of siRNA into targeted tissues, e.g. site of inflammation or cancer, because the RES entraps the siRNA delivery vehicle [37]. On the other hand, both long-circulating and high molecular weight materials are known to accumulate in cancer and inflammatory tissues associated with leaky vasculature histopathological features, a phenomenon known as the EPR effect [9]. Thus, long-circulating particle formulations of siRNA can accumulate in arthritic joints via EPR [40]. A lipid-containing particle suspension of dexamethasone palmitate has been clinically used for the treatment of arthritis in Germany (Lipotalon") and Japan (Limethason"), and its tissue distribution was twice as high in the arthritic joints of rats than that of dexamethasone sodium phosphate. To gain targeted tissue distribution and reduce the liver/spleen distribution of siRNA, recent reports have revealed that modifying targeting molecules such as antibodies [41], peptides [42], aptamers [43], and polysaccharides [44] promoted greater efficiency. Even if targeting molecules are used, delivery efficiency for inflammatory tissue may vary depending on the organ and histopathological features. In the case of cancer treatment with nanoparticles, it is clear that drugs are not distributed in all types of cancer and several barriers prevent cancer tissue penetration [45]. A recent publication compared tissue penetration using 30 to $100 \mathrm{~nm}$ nanoparticles, and showed that only $30 \mathrm{~nm}$ micelles could penetrate poorly permeable tissue to elicit a therapeutic effect [46]. Optimization of the particle size of WS may enhance target tissue distribution and penetration in inflammatory diseases.

The third issue is the off target effects. Non-targeted immune responses cause unexpected side effects or complex therapeutic outcomes. siRNA was shown to be recognized by the toll-like receptor after its systemic administration, which caused a cytokine response [47]. siRNA may not always be specific and can have many off-target 
effects. In the case of nanoparticle delivery, anti-PEG IgM production has been reported after a single intravenous injection of the siRNA encapsulated and PEG-coated lipid-based delivery system [48]. Several studies demonstrated that it was feasible to reduce the toll-like receptor response and off-target effects by a substitution with the 2'-Omethyl modified ribosyl group [47], which also suppressed anti-PEG IgM production in association with PEGylated nanoparticles [48]. Many another chemical modifications and siRNA designing algorithms have recently been reported [33], not only for avoiding immunological responses and off-target effects, but also to obtain robust binding with targeted mRNA [49], an elongated mechanism of action [50]. This modified siRNA and WS delivery system combination may be a promising candidate for highly potent and specific siRNA therapeutics.

\section{Conclusion}

RNAi with improved delivery techniques, such as WS, is a promising new therapy for RA by specific knocking down of the gene of interest.

\section{Acknowledgement}

Nobuhiro Yagi is an employee of Kyowa Hakko Kirin Co., Ltd. Other authors do not have a financial interest that would constitute a conflict of interest. All the authors do not have a financial interest regarding to Lipotalon and Limethason".

\section{References}

1. Scott DL, Wolfe F, Huizinga TW (2010) Rheumatoid arthritis. Lancet 376: 1094-108.

2. Keane J, Gershon S, Wise RP, Mirabile-Levens E, Kasznica J, et al. (2001) Tuberculosis associated with infliximab, a tumor necrosis factor alphaneutralizing agent. N Engl J Med 345: 1098-104.

3. Komano Y, Tanaka M, Nanki T, Koike R, Sakai R, et al. (2011) Incidence and risk factors for serious infection in patients with rheumatoid arthritis treated with tumor necrosis factor inhibitors: a report from the Registry of Japanese Rheumatoid Arthritis Patients for Longterm Safety. J Rheumatol 38: 1258-1264.

4. van Vollenhoven RF (2009) Treatment of rheumatoid arthritis: state of the art, Nat Rev Rheumatol 5: 531-541.

5. Consortium IHG (2004) Finishing the euchromatic sequence of the human genome. Nature 431: 931-945.

6. Meade BR, Dowdy SF (2009) The road to therapeutic RNA interference (RNAi): Tackling the 800 pound siRNA delivery gorilla. Discov Med 8: 253-256.

7. Elbashir SM, Lendeckel W, Tuschl T (2001) RNA interference is mediated by 21- and 22-nucleotide RNAs. Genes Dev 15: 188-200.

8. Zhou HF, Yan H, Pan H, Hou KK, Akk A, et al. (2014) Peptide-siRNA nanocomplexes targeting NF-kappaB subunit p65 suppress nascent experimental arthritis. J Clin Invest 124: 4363-4374.

9. Matsumura Y, Maeda H (1986) A new concept for macromolecular therapeutics in cancer chemotherapy: mechanism of tumoritropic accumulation of proteins and the antitumor agent smancs. Cancer Res 46: 6387-6392.

10. Yagi N, Manabe I, Tottori T, Ishihara A, Ogata F, et al. (2009) A nanoparticle system specifically designed to deliver short interfering RNA inhibits tumor growth in vivo. Cancer Res 69: 6531-6538.

11. Komano Y, Yagi N, Onoue I, Kaneko K, Miyasaka N, et al. (2012) Arthritic joint-targeting small interfering RNA-encapsulated liposome: implication for treatment strategy for rheumatoid arthritis. J Pharmacol Exp Ther 340: 109-113.
12. Khoury M, Escriou V, Courties G, Galy A, Yao R, et al. (2008) Efficient suppression of murine arthritis by combined anticytokine small interfering RNA lipoplexes. Arthritis Rheum 58: 2356-2367.

13. Khoury M, Louis-Plence P, Escriou V, Noel D, Largeau C, et al. (2006) Efficient new cationic liposome formulation for systemic delivery of small interfering RNA silencing tumor necrosis factor alpha in experimental arthritis. Arthritis Rheum 54: 1867-1877.

14. Schiffelers RM, Xu J, Storm G, Woodle MC, Scaria PV (2005) Effects of treatment with small interfering RNA on joint inflammation in mice with collagen-induced arthritis. Arthritis Rheum 52: 1314-1318.

15. Inoue A, Takahashi KA, Mazda O, Terauchi R, Arai Y, et al. (2005) Electro-transfer of small interfering RNA ameliorated arthritis in rats. Biochem Biophys Res Commun 336: 903-908.

16. Firestein GS (2003) Evolving concepts of rheumatoid arthritis. Nature 423: 356-361.

17. Koch AE (2003) Angiogenesis as a target in rheumatoid arthritis. Ann Rheum Dis 62: ii60-ii67.

18. Wang X, Liang J, Koike T, Sun H, Ichikawa T, et al. (2004) Overexpression of human matrix metalloproteinase- 12 enhances the development of inflammatory arthritis in transgenic rabbits. Am J Pathol 165: 1375-1383.

19. Kinne RW, Stuhlmuller B, Burmester GR. (2007) Cells of the synovium in rheumatoid arthritis. Macrophages. Arthritis Res Ther 9: 224.

20. Hammaker D, Sweeney S, Firestein GS (2003) Signal transduction networks in rheumatoid arthritis. Ann Rheum Dis 62: 86-89.

21. Nemeth T, Mocsai A (2012) The role of neutrophils in autoimmune diseases. Immunol Lett 143: 9-19.

22. Mohr W, Westerhellweg H, Wessinghage D (1981) Polymorphonuclear granulocytes in rheumatic tissue destruction. III. an electron microscopic study of PMNs at the pannus-cartilage junction in rheumatoid arthritis. Ann Rheum Dis 40: 396-399.

23. Edwards SW, Hallett MB (1997) Seeing the wood for the trees: the forgotten role of neutrophils in rheumatoid arthritis. Immunol Today 18: 320-324.

24. Korganow AS, Ji H, Mangialaio S, Duchatelle V, Pelanda R, et al. (1999) From systemic $\mathrm{T}$ cell self-reactivity to organ-specific autoimmune disease via immunoglobulins. Immunity 10: 451-461.

25. Wipke BT, Allen PM (2001) Essential role of neutrophils in the initiation and progression of a murine model of rheumatoid arthritis. J Immunol 167: 1601-1608.

26. Ohara M, Saniabadi AR, Kokuma S, Hirata I, Adachi M, et al. (1997) Granulocytapheresis in the treatment of patients with rheumatoid arthritis. Artif Organs 21: 989-994.

27. Sanmarti R, Marsal S, Valverde J, Casado E, Lafuente R, et al. (2005) Adsorptive granulocyte/monocyte apheresis for the treatment of refractory rheumatoid arthritis: an open pilot multicentre trial. Rheumatology (Oxford) 44: 1140-1144.

28. Monach PA, Nigrovic PA, Chen M, Hock H, Lee DM, et al. (2010) Neutrophils in a mouse model of autoantibody-mediated arthritis: critical producers of Fc receptor gamma, the receptor for C5a, and lymphocyte function-associated antigen 1. Arthritis Rheum 62: 753-764.

29. Schulert GS, Grom AA (2015) Pathogenesis of macrophage activation syndrome and potential for cytokine- directed therapies. Annu Rev Med. 66: 145-1459.

30. Lin AM, Rubin CJ, Khandpur R, Wang JY, Riblett M, Yalavarthi S, et al. (2011) Mast cells and neutrophils release IL-17 through extracellular trap formation in psoriasis. J Immunol 187: 490-500.

31. Kallenberg CG, Rarok A, Stegeman CA, Limburg PC (2002) New insights into the pathogenesis of antineutrophil cytoplasmic autoantibodyassociated vasculitis. Autoimmun Rev 1: 61-66.

32. Christensen J, Litherland K, Faller T, van de Kerkhof E, Natt F, et al. (2013) Metabolism Studies of Unformulated Internally [3H]-Labeled Short Interfering RNAs in Mice. Drug Metab Dispos 41: 1211-1219.

33. Deleavey GF, Damha MJ (2012) Designing chemically modified oligonucleotides for targeted gene silencing. Chem Biol 19: 937-954. 
Citation: Komano Y, Yagi N, Nanki T (2015) Joint-Targeting Drug Delivery System for Rheumatoid Arthritis: siRNA Encapsulated Liposome.

Page 5 of 5

34. Nguyen QD, Schachar RA, Nduaka CI, Sperling M, Klamerus KJ, et al. (2012) Evaluation of the siRNA PF-04523655 versus ranibizumab for the treatment of neovascular age-related macular degeneration (MONET Study). Ophthalmology 119: 1867-1873.

35. Molitoris BA, Dagher PC, Sandoval RM, Campos SB, Ashush H, et al. (2009) siRNA targeted to p53 attenuates ischemic and cisplatin-induced acute kidney injury. J Am Soc Nephrol 20: 1754-1764.

36. Gao S, Dagnaes-Hansen F, Nielsen EJ, Wengel J, Besenbacher F, et al. (2009) The effect of chemical modification and nanoparticle formulation on stability and biodistribution of siRNA in mice. Mol Ther 17: 1225-1233.

37. Tavitian B, Marzabal S, Boutet V, Kuhnast B, Terrazzino S, et al. (2002) Characterization of a synthetic anionic vector for oligonucleotide delivery using in vivo whole body dynamic imaging. Pharm Res 19: 367-376.

38. Tabernero J, Shapiro GI, LoRusso PM, Cervantes A, Schwartz GK, et al. (2013) First-in-humans trial of an RNA interference therapeutic targeting VEGF and KSP in cancer patients with liver involvement. Cancer Discov 3: 406-417.

39. Bouchie A. (2012) Companies in footrace to deliver RNAi. Nat Biotechnol 30: 1154-1157.

40. Gabriel D, Lange N, Chobaz-Peclat V, Zuluaga MF, Gurny R, et al. (2012) Thrombin-sensitive dual fluorescence imaging and therapeutic agent for detection and treatment of synovial inflammation in murine rheumatoid arthritis. J Control Release 163: 178-186.

41. Schneider B, Grote M, John M, Haas A, Bramlage B, et al. (2012) Targeted siRNA Delivery and mRNA Knockdown Mediated by Bispecific Digoxigenin-binding Antibodies. Mol Ther Nucleic Acids 1: 46.
42. Toriyabe N, Hayashi Y, Harashima H (2013) The transfection activity of R8-modified nanoparticles and siRNA condensation using $\mathrm{pH}$ sensitive stearylated-octahistidine. Biomaterials 34: 1337-1343.

43. Bagalkot V, Gao X (2011) siRNA-aptamer chimeras on nanoparticles: preserving targeting functionality for effective gene silencing. ACS Nano 5: 8131-8139.

44. Yang F, Huang W, Li Y, Liu S, Jin M, et al. (2013) Anti-tumor effects in mice induced by survivin-targeted siRNA delivered through polysaccharide nanoparticles. Biomaterials 34: 5689-5699.

45. Jain RK, Stylianopoulos T (2010) Delivering nanomedicine to solid tumors. Nat Rev Clin Oncol 7: 653-664.

46. Cabral H, Matsumoto Y, Mizuno K, Chen Q, Murakami M, et al. (2011) Accumulation of sub-100 nm polymeric micelles in poorly permeable tumours depends on size. Nat Nanotechnol 6: 815-823.

47. Whitehead KA, Dahlman JE, Langer RS, Anderson DG (2011) Silencing or stimulation? siRNA delivery and the immune system. Annu Rev Chem Biomol Eng 2: 77-96.

48. Tagami T, Uehara Y, Moriyoshi N, Ishida T, Kiwada H (2011) Anti-PEG IgM production by siRNA encapsulated in a PEGylated lipid nanocarrier is dependent on the sequence of the siRNA. J Control Release 151: 149-154.

49. Elmen J, Thonberg H, Ljungberg K, Frieden M, Westergaard M, et al. (2005) Locked nucleic acid (LNA) mediated improvements in siRNA stability and functionality. Nucleic Acids Res 33: 439-447.

50. Wada S, Obika S, Shibata MA, Yamamoto T, Nakatani M, et al. (2012) Development of a 2',4'-BNA/LNA-based siRNA for Dyslipidemia and Assessment of the Effects of Its Chemical Modifications In vivo. Mol Ther Nucleic Acids 1: 45. 